\title{
Regulatory requirements and ICH guidelines on carcinogenicity testing of pharmaceuticals: A review on current status
}

\author{
G. B. Jena, C. L. Kaul, P. Ramarao
}

Department of
Pharmacology and
Toxicology, National Institute
of Pharmaceutical Education
and Research, Sector-67,
S.A.S. Nagar,
Punjab-160062, India.
Received: 25.6.2004
Revised: 22.12.2004
Accepted: 4.1.2005
Correspondence to:
Dr. P . Ramarao
E-mail:
ramaraop@yahoo.com

\begin{abstract}
International regulatory guidelines require that new chemical entities (NCEs) be tested first on animals (preclinical studies) for safety and efficacy before the start of clinical development. Long-term nonclinical studies such as carcinogenicity testing, the subject of the present review, is conducted in tandem with on-going clinical studies. Over the last few years, a number of regulatory changes have occurred in the regulatory requirements of carcinogenicity testing and the use of such data in human risk assessment process. The efforts are to provide a better mechanistic basis in interpretation of standard lifetime and short-term rodent bioassays. The major areas of focus of this review include the basic considerations for the lifetime rodent bioassay, the recommendations of the International Conference on Harmonization (ICH), and the development and validation of new short-term or alternative models for carcinogenicity testing. The objectives of these guidelines are to avoid the unnecessary use of animals in testing and to provide consistency in world wide regulatory assessments of applications. It will ultimately improve the precision in carcinogenicity testing, minimize the time requirement for such testing, and help to conserve the resources in the process of drug discovery and development.
\end{abstract}

KEY WORDS: Carcinogenicity bioassay, cancer risk assessment, drug safety, cancer chemotherapy, preclinical toxicity tests, transgenic models.

\section{Introduction}

Carcinogenicity studies are conducted in laboratory animals to evaluate the carcinogenic potential of pharmaceuticals. ${ }^{[1,2]}$ In drug discovery, rodent carcinogenicity studies are conducted with an objective to explore the mechanism of carcinogenesis and the ultimate risk to humans. ${ }^{[3]}$ Many problems are encountered in carrying out and interpretation of results for carcinogenicity bioassay. A number of factors influence the results of a carcinogenicity study. ${ }^{[4]}$ Animal carcinogenicity studies are still considered as the best approach in predicting human risk assessment. ${ }^{[5]}$ Regulatory approval of a new chemical entity (NCE) is primarily based on the results obtained from long-term studies in rats and mice. ${ }^{[6]}$ The classical carcinogenesis studies refer only to the strength of the experimental evidence and not to identify the potency and mechanism. Therefore, it increases the chances of falsepositive and false-negative results. However, many previously marketed drugs have exhibited carcinogenic potential and have raised serious public concern when detected by the new/ alternative methodologies. ${ }^{[7]}$ Over the last two decades, carcinogenic risk- estimation process has progressed rapidly, resulting in the incorporation of many new regulations with the objective of protecting human health from chemical hazards. ${ }^{[8]}$ Several methods are being used to screen these agents at preclinical levels. ${ }^{[9]}$ Development of new 'short-term tests' based on the understanding of mechanism of action would help in the set up of batteries of such tests and tier-testing schemes that would greatly assist in scientific and regulatory decision making. To explore the further possibilities and to improve the precision in predicting carcinogenic risk to humans, constant review of methodologies and broader agreement on procedure at global level are needed. In this review an attempt has been made to discuss the (i) current status of carcinogenesis testing procedures, (ii) the new ICH guidelines, and the (iii) influence of new technologies/alternative test models in the assessment of carcinogenic potential of new drugs.

\section{Assessment of human cancer risk: Challenges and regulations}

The Carcinogenesis process

Cancer development is a multistage process in which the summation of event is required to produce a malignant tumour. ${ }^{[10],[11]}$ The process starts with the initiation phase, followed by the promotion phase, and finally by the progression 
phase. The initiation phase is an irreversible consequence of the interaction of a tissue with a carcinogen, which is not recognized as a pathological entity, but produces cells that are precursors to the tumour. Promotion is defined as a reversible process that facilitates the expression of initiated phenotype. Promotion is distinguished from progression, where the former has its influence at the tissue level, whereas the later represents further phenotypic alteration in initiated cells. The conversion from pre-malignant phenotype to a malignant cell type is recognized as the major time-dependent stage in cancer pathogenesis. ${ }^{[12]}$ However, tumour heterogeneity associated with the acquisition of metastasis potential is a relatively rapid event. The evaluation of the relations between mutagenicity, genotoxicity, and carcinogenicity studied in different in vitro models indicate that the origin of cancer is in a single cell, and that somatic mutation plays a major role. ${ }^{[13],[14]}$ Multiple genomic alterations are involved in the development of most human cancers. They include alterations in oncogenes, tumour suppressor genes, DNA mismatch repair, and excisionrepair genes. ${ }^{[15]}$ Studies on the pathways for metabolic activation and detoxification of carcinogens and macromolecular interaction have led to the development of methods to detect carcinogen-macromolecule adducts in human population and the emergence of DNA as a critical target site for carcinogens. ${ }^{[16]}$

Carcinogenicity testing procedures used in regulatory toxicity

In the process of drug development, pharmaceuticals are routinely tested for safety and efficacy before they are being marketed. The financial investment in drug discovery grows exponentially as the compound progresses from initial discovery, through development to marketing. ${ }^{[17], ~[18] ~ T h e ~}$ primary objective of drug safety evaluation is to obtain biological information that is indicative of toxicity, which can be interpreted and/or extended to the assessment of health risk to humans. ${ }^{[9], ~[19]}$ In early days, reasonably well-defined chronic bioassays in rats and mice were developed to identify carcinogenic potential of human pharmaceuticals. ${ }^{[20], ~[21] ~ T h e s e ~}$ tests are needed for a compound that needs to be administered continuously or intermittently over a prolonged period. In addition, compounds with a mutagenic potential or a structural alert indicating a putative carcinogenic effect, or influence on cell proliferation have to be tested, even if it is intended for a short period of administration. The requirement of carcinogenicity data for the conduct of clinical trials and the final marketing of the compound depend on the guidelines of different regulatory authorities. ${ }^{[22]}$ In spite of various in vivo and in vitro assays designed to detect different endpoints, animal carcinogenicity studies, despite their in-built limitations are said to be the best way of predicting the carcinogenic potential of pharmaceuticals.

\section{The paradigm shift and regulatory requirement}

Depending upon the nature of the therapeutic agent and current paradigms of safety and efficiency evaluation, the preclinical animal toxicological data are considered on a caseby-case basis to optimize and expedite drug development process. Toxicity assessment of a new drug molecule can be performed early in the evaluation process in parallel with pharmacological assessment. Toxicity information provided early in the developmental stages can substantially reduce the financial expenditure through the reduction of attritions. Preclinical studies are limited at the beginning of clinical development. Long-term nonclinical testing (e.g., repeat dose toxicity, genotoxicity, reproductive toxicity, immunotoxicity, carcinogenicity studies) is conducted in parallel with the ongoing clinical studies. The extent of nonclinical studies to be conducted to support the various stages of clinical development differs among nations. It is generally agreed that the highest possible standards of studies are needed, because these studies are time consuming, expensive, requires large number of animals, and often difficult to interpret in terms of human relevance. All guidelines allow some degree of flexibility on the basis of scientific justification. Because the future of an important new therapy rests upon the correct conduct of a toxicity study and its evaluated interpretation, it is essential to observe Good Laboratory Practice (GLP) during the entire study period. ${ }^{\text {23], [24] }}$

\section{Role of National and International regulatory bodies}

Most of the countries are having the specific guidelines for testing of pharmaceuticals for carcinogenicity. In India, Schedule ' $\mathrm{Y}$ ' of Drugs and Cosmetics Rules 1988 issued by the New Drugs Division of Central Drug Standard Control Organisation (CDSCO), Directorate General of Health Services (DGHS), Ministry of Health and Family Welfare, Govt. of India, deals with the prerequisites to carry out the clinical trials of a new drug before its marketing, depending upon the status of the drug in other countries. It is currently being under revision and expected to be released shortly. As per the regulatory requirements, carcinogenicity testing is needed, when a compound or its metabolite is structurally related to a known carcinogen, or when the nature and action of the drug(s) suggest a mutagenic/carcinogenic potential. ${ }^{[25]}$ In the United States, the FDA (Food and Drug Administration) and Centers for both Drugs/Biologics Evaluation and Research (CDER and CBER, respectively) recommend carcinogenicity testing for all new drugs. ${ }^{[5]}$ In the United States, carcinogenicity studies are needed for most compounds being marketed, and when a cause of concern for clinical trials (for details vide infra) exists. In the European Community (EC), carcinogenicity data are required for marketing authorization. ${ }^{[26]}$ In Japan, the Ministry of Health and Welfare (MHW) adopted carcinogenicity tests in 1989 as one of the several toxicity studies required for the approval to manufacture or import of new drugs. ${ }^{[27]}$ These guidelines reflect the complexities of lifetime carcinogenesis bioassay and uncertainties over conducting the ideal study. The International Agency for Research on Cancer (IARC) regularly reviews and evaluates the most commonly used drugs for carcinogenicity on the basis of genotoxicological and epidemiological data in humans. ${ }^{[28], ~[29] ~}$ It provides a reasonably comprehensive catalog of chemicals at various stages of testing that should be consulted before undertaking time-consuming and costly long-term carcinogenesis experiments.

The transgenic mouse models (discussed in detail later) used in carcinogenesis bioassay originally started as independent activities in Japan, USA, and Europe. In the mid 1990s, these models were developed into a more collaborative research effort and supported by the international regulatory agencies in the field of drug safety testing. Later on, in 1996 
an extensive collaborative international research program was initiated and coordinated by the Health and Environmental Sciences Institute (HESI) branch of the International Life Sciences Institute (ILSI) to harmonize and provide the framework for an adequate and systemic evaluation. ${ }^{[30]}$ As the focus of the ICH discussion centered on pharmaceuticals, the collaborative efforts with ILSI-HESI also dealt with pharmaceuticals. [Table 1] The main objective of the collaboration was to generate the scientific data that is needed to understand the benefits and limitations of new carcinogenicity-testing methods, and to assess their ability to add relevant information for predicting human cancer risks. Several chemicals which are genotoxic, non-genotoxic, carcinogenic, and noncarcinogenic were evaluated using the animal models that included the p53+/- knockout mouse, the rasH2 transgenic mouse, Tg.AC transgenic mouse (dermal and oral administration), the homozygous XPA knockout, and the neonatal mouse models. ${ }^{[31-[35]}$ These studies suggest that some of these models could be used in conjunction with information from other sources in a weight of evidence for the integrated analysis approach to risk assessment.

The genesis and concept of ICH process

The ICH harmonization process was first started in 1991 in Brussels, Germany. It is a tripartite agreement between the European Community (EC), USA, and Japan on the issues related to the technical requirements for developing and registering products containing new drug substances. It was started from the long-drawn efforts of the International Association of Pharmaceutical Technology (IAPT) to develop uniform guidelines and to involve all the major countries to agree on a single common approval for new drugs. The main objectives of ICH are the identification and elimination of the duplicate studies and to meet different regulatory requirements, which results in the more efficient use of resources in the research and development process. The other objective is to bring different agencies to one platform with a common purpose and to provide high quality, safe, and effective medicines to patients within a stipulated duration of time (http://www. Ifpma.org/ich1.html).

In parallel with scientific advancements, the ICH Safety Working Group started to evaluate and harmonize the new ICH Guidance on 'Testing for Carcinogenic Potential for Pharmaceuticals'. The basic principle of the new approach is to improve the carcinogenic risk assessment process in terms of human relevance and reduction of both animal and financial resources. It encourages the use of new advanced techniques in assessment process and to foster the development of better alternative methods for future use. It also ensures that all the information should be integrated in the carcinogenic risk assessment process by improving the understanding of the real cancer risks for patients who receive treatment with pharmaceuticals. ${ }^{[36]}$

\section{The ICH guidelines: A new approach}

Carcinogenicity studies are generally conducted to identify a tumorigenic potential of a drug in animals and to assess the relevant risk in humans. The guidelines issued by the ICH covers all pharmaceutical agents including biotechnology-derived pharmaceuticals, which need carcinogenic testing before regulatory approval. It outlines factors of importance to
Table 1

Compounds assessed for carcinogenicity through HESI-ILSI collaborative research programme

\begin{tabular}{|c|c|}
\hline Properties & Compounds \\
\hline Genotoxic human carcinogens & $\begin{array}{l}\text { Cyclophosphamide, } \\
\text { Melphalan and Phenacetin }\end{array}$ \\
\hline $\begin{array}{l}\text { Immunosuppressant human } \\
\text { carcinogens }\end{array}$ & Cyclosporin A \\
\hline Hormonal carcinogens & Diethylstibestrol and Estradiol \\
\hline $\begin{array}{l}\text { Rodent carcinogens/putative } \\
\text { human non carcinogens } \\
\text { (based on human data) }\end{array}$ & $\begin{array}{l}\text { Phenobarbital, Clofibrate, } \\
\text { Reserpine, Dieldrin and } \\
\text { Methapyrilene }\end{array}$ \\
\hline $\begin{array}{l}\text { Rodent carcinogens/ putative } \\
\text { human noncarcinogens } \\
\text { (by mechanism) }\end{array}$ & $\begin{array}{l}\text { Haloperidol, Chlorpromazine, } \\
\text { Chloroform, Metaproterenol, } \\
\text { WY-14643[4-chloro-6-(2,3- } \\
\text { xylidino)-2-pyrimidinylthio], } \\
\text { DEHP(Di(2-ethylhexyl) } \\
\text { phthalate) and Sulfamethoxazole }\end{array}$ \\
\hline Noncarcinogens & $\begin{array}{l}\text { Ampicillin, D-mannitol and } \\
\text { Sulfisoxazole }\end{array}$ \\
\hline
\end{tabular}

consider in choosing the species for the standard 2-year bioassay. With careful retrospective evaluation of several databases, it was found that the data from second species (usually mouse) gave very little information for a definitive regulatory decision of the compound..$^{[37 \mid-39]}$ On the basis of the number of considerations discussed in the guidance, the rat is often being the preferred species for the standard 2-year carcinogenicity assay. One of the most important changes in the new guideline is the ability to use alternative in vivo models (see section 6.0) for carcinogenicity testing in place of 2-year bioassay in mice. Importantly, the alternative models should provide information that is not readily available from the standard 2-year assay in rat, and results should address issues of concern that are relevant to the particular pharmaceutical. Furthermore, the ICH guidelines highlight the totality of diverse studies that provide information about the mechanism of tumour formation in response to treatment with a specific pharmaceutical.

The following three ICH guidelines on carcinogenicity studies of pharmaceuticals have been issued:

S1A: Guideline on the need for carcinogenicity studies of pharmaceuticals, March 1996.

This guideline, in summary, defines the need and conditions under which carcinogenicity studies should be conducted to avoid the unnecessary use of animals in testing. ${ }^{40]}$

$S 1 B$ : Testing for carcinogenicity of pharmaceuticals, July 1997.

This guideline provides methods of experimental approaches for the evaluation of carcinogenic potential of pharmaceuticals. This also obviates the necessity for the routine conduct of two long-term rodent carcinogenicity bioassays; whether the use of rats or mice alone would result in the loss of information on carcinogenicity relevant to human risk assessment has been addressed in this guidance. ${ }^{[1]}$

S1C: Dose selection for carcinogenicity studies of pharmaceuticals, March 1995. 
$S 1 C(R)$ : Addendum to dose selection for carcinogenicity studies of pharmaceuticals: Addition of a limit dose and related notes, July 1997

These guidelines pertain to issues to consider in selecting doses for the conduct of dose range-finding and 2-year studies.

Additionally, the Center for Drug Evaluation and Research of US FDA in May 2002, has issued guidance on "Carcinogenicity Protocol submissions". This is intended to inform drug candidate sponsor of the types of information the FDA relies on when evaluating study protocols for animal carcinogenicity studies.

\section{Carcinogenicity testing: Experimental approach}

\section{Objectives}

Carcinogenicity studies are generally conducted to identify a tumorigenic potential of the drug in animals and to assess the relevant risk in humans. Carcinogenesis is a complex process and no single experimental approach can be expected to predict accurately the carcinogenic potential of a chemical in humans. The basic scheme comprises one long-term (life span) rodent carcinogenicity study plus one other long or shortterm study, which provides additional information that is not readily available from the first long-term study. There are several reasons for choosing a short- or medium-term test system as supplement to the 2-year bioassay. These models can provide better answers for the differences in the range of susceptible target tissues in which tumour develop, and the knowledge of compound's ADME (Absorption, Distribution, Metabolism and Excretion) profile for human risk assessment.

\section{Factors to consider}

\section{Drug candidates}

Pharmaceuticals, which are expected for continuous use or at least for 6-months clinical use, should be considered for carcinogenicity testing. For pharmaceuticals used repeatedly in an intermittent manner in the treatment of chronic or recurrent conditions, carcinogenicity studies are generally needed. It may be noted that most of the pharmaceuticals indicated for 3-months treatment would also likely be used for 6 months or more. Pharmaceuticals administered infrequently or for short duration of exposure (e.g., anesthetics, critical care medicines, radiolabeled imaging agents, and diagnostic aids) do not need carcinogenicity studies unless there is cause for concern (see subsequent text). ${ }^{[3]}$

Compounds for which carcinogenicity studies are needed should be completed before filing an application (e.g., NDA) for marketing approval. However, completed rodent carcinogenicity studies are not needed in advance of the conduct of large-scale clinical trials, unless there is a special concern for the patient population. ${ }^{[37]}$ For pharmaceuticals intended to treat life-threatening or severely debilitating diseases (e.g., AIDS), carcinogenicity testing need not be conducted before market approval, and studies should be conducted post-approval. If the life expectancy in the indicated population is short (i.e., less than 2-3 years) no long- term carcinogenicity studies may be required. If the therapeutic agent is clinically successful and can be used in non-cancer situations, and life is considerably increased after the treatment, then carcinogenicity studies are generally needed. ${ }^{[3]}$ Certain formulations or delivery systems, which may result in prolonged exposures, need carcinogenicity testing. Carcinogenicity studies are not generally needed for endogenous substances. Endogenous peptides, proteins and their analogs produced by chemical synthesis, by extraction/ purification from an animal/human source or by biotechnological methods such as recombinant DNA technology may require special consideration. Although not usually necessary, under certain circumstances such as the treatment duration, clinical indication, or patient population long-term carcinogenicity studies in rodent species should be considered. ${ }^{[42]}$

Cause for concern

Some pharmaceuticals should be tested for carcinogenicity if there is concern about their carcinogenic potential. Several factors which could be considered may include: (i) previous demonstration of carcinogenic potential in the product class which is relevant to human (class alert); (ii) structure-activity relation suggesting carcinogenic risk; (iii) evidence of preneoplastic lesions in repeated dose toxicity studies; and (iv) long-term tissue retention of parent compound or metabolite(s) resulting in local tissue reactions or other pathophysiological responses. ${ }^{[43]}$

\section{Genotoxicity}

Pharmaceutical databases are being used to determine the association between genetic toxicology test findings and rodent carcinogenicity outcomes. Sometimes chemical-induced carcinogenesis may involve a non-genotoxic mechanism. So it is difficult to determine how well genetic toxicology assays predict carcinogenic potential. Indomethacin tested negative for in vivo cytogenetic assays in the regulatory tests, but was reported positive for the induction of DNA adducts in the literature. Halothane and pyrazinamide were also in vivo positive for comet test in human lymphocytes and induction of sperm head abnormalities in mice, respectively, which are considered non-regulatory tests. ${ }^{[7]}$ The importance of these positive findings in the relatively insensitive rodent cytogenetic assays is unclear. All the intrinsic values and limitations of different test systems should be taken into account to reduce the risk of false-negative results for compounds with genotoxic potential. ${ }^{[4]}$ At the same time, a single positive result in any assay for genotoxicity does not necessarily mean that the test compound poses a genotoxic hazard to humans. ${ }^{[45]}$ In such a case, mechanistic investigations can result in further details that will aid in taking a regulatory decision.

\section{Experimental design}

Two carcinogenicity studies have been recognized: (i) one long-term carcinogenicity study in the rat, a species of choice; (ii) an additional in vivo study that supplements standard 2year rat study. This is either a long-term carcinogenicity study in a second rodent species, mouse, or short- or medium-term in vivo rodent test systems. The later is discussed in section "Short or medium-term (alternative) models in carcinogenicity bioassay”.

Role of study protocol in designing carcinogenicity studies

The study protocol for a definitive study is largely based on scientific reasoning, experience, and consensus gained over many studies. Parameters such as the number of animals, the adequacy of dose, duration and the gross histopathological examination are considered for a particular study. Several 
important factors such as species, strain, sex, dose levels, test substance purity, route of administration, and statistical methods are considered in designing a 2-year carcinogenicity study. Other factors to consider are pharmacology, toxicology and metabolism of the drug, and systemic exposure (e.g., as measured by AUC) achieved in the test species as multiples of maximum recommended human dose (MRHD). On the basis of a number of considerations and the extensive database available on tumors, it is considered that the rat is the most preferred species for the standard 2- year carcinogenicity bioassay. ${ }^{[46]}$

Because a large number of animals and length of time is involved, it is essential that these studies be planned well. Inadequate dosing will result in improper results and may lead to the necessity of repeating these studies. To avoid such problems, the US FDA strongly recommends the sponsor to conduct a 90-day dose range-finding study and submit the results along with the protocol for the carcinogenicity study to the referred division for comment. The intention of this protocol is to inform the drug candidate sponsors about the types of information the agency relies on when evaluating protocols for animal carcinogenicity studies ${ }^{[4]}$ (http://www.fda.gov/cder/ guidance/index.htm).

Species and strain selection

The lifetime (2 year) carcinogenic bioassays are generally conducted in both sexes of rats and/or mice. In most studies, inbred rodent strains are used in order to reduce experimental variables and to enhance the interpretability of the results. The control of experimental variables and the large experience with common laboratory strains of rats and mice represent great advantages for risk assessment. Background data such as food intake, body weight, growth rate, longevity, clinical pathology, and histopathology can facilitate the interpretation of experimental results. Historical data on tumour incidence is considered useful in the interpretation of long-term rodent carcinogenicity bioassays, especially to assess the occurrence of marginally increased tumour incidence and species differences. ${ }^{[48]}$ These data are helpful to avoid the high incidence of background tumors and aging lesions that arise spontaneously in different organs in different strains of rats and mice. As these data are compared with the treated animals, the concurrent control group is considered to be the most critical parameter. ${ }^{[49]}$

To draw a definite conclusion whether a test compound can be evaluated on a particular species, information on genetic toxicology, tumour incidence, strain of animal, route and dosage regimen, pharmacological or therapeutic activity, development and/or regulatory status, and relevant reason for termination of development are generally considered. Compounds that induce tumors only in a single species are approximately double in number in rats as compared to mice. ${ }^{[38 \mid}$ In a simplistic sense, this implies that the rat is more "sensitive" than the mouse. There are a few instances identified in which mouse tumors are considered as the sole reason for the regulatory action concerning disapproval for marketing of a pharmaceutical. ${ }^{[37]}$ Rodent liver is highly susceptible for the induction of tumors by non-genotoxic chemicals. These tumors are not always relevant to carcinogenic risk in humans and mislead the use of rodent for carcinogenic risk estimation. The rat carcinogenicity study is widely accepted because these results can correlate up to $70 \%$ with that of human data. ${ }^{[50]}$

Non-genotoxic chemicals induce carcinogenicity in rodents, which is highly dependent on species, strain, and target organ. The induction of carcinogenicity depends on the threshold dose phenomenon and it varies from species to species. Mechanistic studies have permitted the distinction between effects that are specific to the rodent model and those that are likely to have relevance for humans. The specific role of tissue specificity of receptors and their subtypes has increased our understanding of the mechanistic basis of carcinogenesis. ${ }^{[51]}$

On the basis of metabolic pathways, neither rats nor mice are considered suitable for the conduct of the bioassay. ${ }^{[52]}$ Much attention is now being paid to the pharmacokineticpharmacodynamic relation. Rapid progress is being made on the specific role of cytochrome P450 isozymes mediated biotransformation of drugs. Because most of the research activity is confined to rats and humans, the data obtained from mice would be less likely to provide useful metabolic information in mechanistic studies. ${ }^{[53]}$ Owing to the size of the animal, the mouse model is not considered suitable when it comes to the collection of serial blood samples, microsurgery/ catheterization, and organ weights. Animal sacrifice is required to collect blood samples; hence more numbers are required per investigation. ${ }^{[50]}$

On the basis of the availability of historical control database on tumors, the following strains are commonly used. Rats: Fischer 344, Sprague-Dawley, Wistar. Mouse: B6C3F1, ICR Swiss (CD-1), BALB/c. In a recent study, Britton et al (2004) reports that of the three rat strains studied (Harlan Hsd:Sprague-Dawley SD, Harlan Wistar Hsd:BrlHan:WIST, Charles River Crl:CD), Harlan Wistar strain survived in much greater numbers in 104-week carcinogenicity study. ${ }^{[54]}$ The improved survival rate, according to the authors, appeared to be independent of body weight and food consumption and is reflected in the spontaneous pathology profile.

It is now well recognized that the diet fed ad libitum can directly enhance weight gain resulting in obesity, increased background tumour development, and non-neoplastic lesions. Owing to these undesirable trends, the longevity of the animals decline, resulting in 50\% survival level for some strains of rats before the 24-months target point. Alternative diets and feeding regimens are being evaluated and some results have already been published. ${ }^{[5]}$ There are many options available for ad libitum feeding and changes in the dietary content need to be controlled within a study for proper biological and statistical assessment of neoplastic and non-neoplastic lesions.

\section{Group number and size}

Generally, for biological and statistical determinations, three treated groups are recommended for establishing any dose-related effects. The conventional number of animals per sex per group is 50 to 100 . In many studies, $50 \%$ or more survivors, over 18 months for mice and 24 months for rats are observed. This survival rate is considered acceptable for detecting age-related, non-lethal conditions and for statistical analysis. ${ }^{[56],}$ [57] The scientific reason for using more animals per group is to increase the statistical power of the study. ${ }^{[33]}$ Sometimes the control group is doubled to include two concurrent groups with 50 animals per sex per group that 
generates a large histopathological database. Two control groups are used in cases such as dietary administration where control 1 group is given diet/vehicle ad libitum, and in control 2 group, diet is restricted to the amount consumed by the highdose group. In some studies, 3 control groups are used: 2 diet controls (each kept in two different rooms) and 1 vehicle control. For dose range-finding study, 10 to 30 animals per sex per group are used. Satellite groups each composed of 10 animals per sex per group (at least 2 animals/sex/time point) are used for toxicokinetic measurements.

\section{Route of administration}

The route of exposure in animals should be the same as the intended clinical route. If similar metabolism and systemic exposure can be observed through different routes of administration, then carcinogenicity studies should be conducted by using a single route. The target organ for the clinical route should be adequately exposed to the test material. The evidence of adequate target-organ exposure should be obtained from toxicokinetic data. ${ }^{58]}$ The conventional route of administration is oral by gavage or dietary administration.

Pharmaceuticals applied topically with poor systemic absorption may not need studies by the oral route to assess the carcinogenic effect to internal organs. However, in case of photocarcinogens, dermal application (generally in mice) may be needed. For different salts of the same base, where prior carcinogenicity studies are available, evidence of changes in pharmacokinetics, pharmacodynamics, or toxicity should be provided. When changes in exposure and consequently toxicity are noted with a change in salt or combination with another drug molecule (e.g., fixed dose combination), then additional studies are recommended to determine the carcinogenic potential of the test substance under investigation. ${ }^{[59]}$

Duration of study

There is a general agreement upon the exposure time of 18 to 24 months for mice and at least 24 months for rats in lifetime carcinogenesis bioassay. Both the time frames agree well for inducing tumors because of continuous administration of test chemical. Extending the study period beyond this time limit, especially for a negative result and using the $50 \%$ survivor rule for termination of the study will further increase confidence in test results obtained. ${ }^{[3]}$ The duration for dose range-finding study is 90 days if it is intended to support dose selection for a standard 2-year study. The duration will be 4 week in a wildtype strain if it is intended to support a short-term study.

Issues for the conduct of dose-ranging studies

The 90-day dose range-finding study is initiated to select the high dose (as well as middle and low doses) for the definitive 2-year carcinogenesis bioassay. The metabolic profiles of the drug in humans and the animal species under investigation are provided for the assessment of its carcinogenic potential. It is unnecessary to include the maximum feasible doses in the design of a range-finding study, when a dose lower than the maximum feasible dose administered by the same route of administration is not tolerated or exceed other acceptable dose selection endpoints. In the absence of this information, it may be prudent to include a maximum feasible dose in the design of a range-finding experiment. The maximum dose selected should not result in death or induce any significant toxicity in the tested animals.

\section{Dose selection}

Owing to the diverse nature of substances used in pharmaceuticals and many non-genotoxic mechanisms involved in carcinogenesis, a flexible approach is needed for dose selection. However, in the process of defining such a flexible approach, it is recognized that the fundamental mechanisms of carcinogenesis are only poorly understood at the present time. The plasma concentration of drug substances represents an important parameter in selecting a particular dose and improving the design of the rodent bioassay. Consideration of other relevant animal toxicity data and integration with available human data is paramount in selecting the high dose for the carcinogenicity study. ${ }^{[60]}$

The selection of appropriate dose levels is of paramount importance for the successful outcome of any study. Dose selection requires an in-depth knowledge of a compound's pharmacology, repeat dose toxicology, toxicokinetics in both test species, and pharmacokinetics in humans. It is generally agreed upon by all regulatory authorities to set a high dose, which induces some type of toxicity, which is not life threatening for the study period. The best known example is a $10 \%$ reduction in body weight gain relative to control. In case of a test substance, which is nontoxic or has a low bioavailability, multi-dose toxicokinetic measurements through different routes should permit in the selection of a route and a dose for maximum exposure. Another alternative but still an arbitrary approach is to determine high multiples of the human dose (30 to 100 times) expressed as an area under the curve (AUC). Such high doses may not be achievable for toxic compounds in rodents. ${ }^{[61]}$

Several methods are being used in the selection of doses for range-finding experiments. There is no uniformity among regulatory agencies around the world. For example, the maximum tolerated dose (MTD) has been used in the United States, whereas Europe and Japan normally select the high dose on the basis of toxicity endpoints. Some countries resort to high multiples of the MRHD. The chosen doses should clearly elicit effects that can be used as endpoints as recommended in the ICH guidance. Additionally, the study should also include a dose that is without significant toxicity. ${ }^{[62]}$ There are as many as six different methods for selection of the high dose for rangefinding experiments.

a) Toxicity-based endpoints

The ICH Expert Working Group on Safety has agreed to use the maximum tolerated dose (MTD) as an acceptable toxicitybased endpoint for selection of the high dose for carcinogenicity studies. ${ }^{[63]}$ The MTD is predicted to produce a minimum toxic effect or the maximum dose that is being tolerated over the course of the study. This type of effect can be predicted from a 90-day dose range-finding study in which minimal toxicity can be observed. Factors such as alteration in physiological function, no more than $10 \%$ decrease in body weight gain relative to control, target-organ toxicity, and significant alterations in clinical pathological parameters are considered for high dose selection. It is important that the maximum dose selected should allow an adequate margin of safety, not to significantly disturb physiological function of the animal, and 
finally good survival till the end of the dosing period.

\section{b) Pharmacokinetic endpoints}

The dose administered to different species may not correspond to tissue concentrations because of different metabolic and excretory patterns. The unbound drug in plasma is thought to be the most relevant indirect measure of tissue concentrations of the drug. The AUC is considered to be the most comprehensive pharmacokinetic endpoint, because it takes into account the plasma concentration of the test substance and the in vivo retention time. ${ }^{[64]}$ For non-genotoxic pharmaceuticals, a systemic exposure representing large multiples of the human AUC (at the maximum recommended daily dose) may be an appropriate endpoint for dose selection. The selection of a high dose for carcinogenicity studies, which represents systemic exposure ratio of 25 -fold rodent to human plasma AUC of parent compound and/or metabolites, is considered pragmatic. For example, systemic exposure ratio of 25-fold, rat to human MRHD based on body surface area $\left(\mathrm{mg} / \mathrm{m}^{2}\right)$ would be $25 \times 37$ (human) / 6 (rat) = approximately 150 -fold $\mathrm{mg} / \mathrm{kg}$ ratio. Therefore, a human dose of $500 \mathrm{mg} /$ day or less could be tested in rats at $1500 \mathrm{mg} / \mathrm{kg} /$ day as the high dose. ${ }^{[60]}$

\section{c) Saturation of absorption}

The measurement of saturation of absorption of drugrelated substances from systemic circulation are an acceptable method in high dose selection. The low and mid doses are selected on the basis of the saturation of metabolic and elimination pathways of the compound under investigation. ${ }^{[65]}$

\section{d) Pharmacodynamic endpoints}

Pharmacodynamic endpoints for high dose selection are compound specific and are considered for a particular study on the basis of scientific merits. The high dose selected should produce a pharmacodynamic response in animals of such a magnitude that would preclude further dose escalation. Meanwhile, this dose should not disturb normal physiology or homeostasis of animals (e.g., causing hypotension) ${ }^{[66]}$

\section{e) Maximum feasible dose}

The use of pharmacokinetic endpoints (AUC ratio) in dose selection for low-toxicity pharmaceuticals can considerably decrease the need for selecting high doses on the basis of feasibility criteria. However in certain conditions, neither toxicity-based nor a pharmacodynamic-based dose selection can be achieved, and determination of pharmacokinetic endpoints (the 25 -fold $\mathrm{mg} / \mathrm{m}^{2}$ ratio of rodent to human AUC or saturation of absorption) is also not feasible. For non-genotoxic pharmaceuticals in a long-term carcinogenicity testing, a limited dose of up to a maximum of $1500 \mathrm{mg} / \mathrm{kg} /$ day in rats is considered acceptable where the MRHD is approximately 500 $\mathrm{mg} /$ day. The maximum feasible dose by dietary administration is considered $5 \%$ of total diet. In addition to dietary administration, when other routes are followed the high dose will be limited and considered on the basis of practicality and local tolerance. ${ }^{|67|}$

\section{f) Additional endpoints}

Additional endpoints can be used on high-dose selection for rodents on the basis of scientific rationale, which has not been defined in the above guidelines. Such designs are evaluated on the basis of their individual merits for a specific pharmaceutical compound. Mechanisms such as cell prolif- eration, induction of apoptosis, and epigenetic factors that play a crucial role in the induction of carcinogenesis, should be considered in high-dose selection for carcinogenicity studies. ${ }^{[68]}$

The mid and low doses provide information that would assist in assessing the relevance of study findings to humans. These doses are selected following integration of rodent and human pharmacokinetics, pharmacodynamic, and toxicity data. Some of the factors to be considered while selecting middle and low doses are: linearity of pharmacokinetics and saturation of metabolic pathways, human exposure and therapeutic dose, pharmacodynamic response in rodents, mechanistic information and potential for threshold effects, and alterations in normal rodent physiology. ${ }^{[69]}$

\section{observations and measurements}

All animals should be observed for clinical signs and mortality daily throughout the study. Palpation to examine for nodule formation is conducted frequently. Body weight and food consumption are recorded weekly. Hematology and clinical chemistry parameters are determined once, preferably at the end of the study. However, most of the investigators consider them optional. For toxicokinetics study, blood samples are collected from the caudal vein in unanaesthetized state from satellite animals (5 to 10/ sex/group) at various intervals such as 4, 12, 26, 52 and 103 weeks (morning and afternoon). The satellite animals are usually sacrificed after final sampling without further investigation. At the end, all surviving animals should be subjected to detailed necropsy that includes weighing and histopathological examination of several organs and tissues from control and high dose groups, and for any animal that died or was moribund and sacrificed.

The organs evaluated for histopathology are very exhaustive and are in general, and those organs that are most likely to develop cancer should be evaluated for all animals in all groups. The frequently recommended organs are liver, kidney, adrenal, uterus, and GI tract. Additional organs can be added to monitor a potential target-organ effect. When high-dose effects are observed in the target organs a limited number of tissues are recommended. The interpretation of results from the study will be dependent upon the list of tissues evaluated and the supportive data obtained from repeat dose toxicology studies. Any organ that does not have a human counter part, for example, zymbal glands, should generally be excluded from the study. The type of pathological changes could be either positive neoplastic or non-neoplastic lesions evolved due to either known secondary mechanisms or may be caused by study factors such as dietary intake.

Additional investigations are generally encouraged in all guidelines for better interpretation of the results. It has been recommended that blood smears and organ weights (e.g., adrenals, brain, heart, kidneys, liver, lungs, ovaries, pituitary, spleen, testes, and uterus) should be carried out at the time of termination. Smears of adequate quality, usually from terminal animals, are used in confirming specific granulocytic leukemia in Sprague Dawley rats that have histopathological evidence of such lesions. The organ weights are questionable for organs from a few animals that are distorted in weight due to lesions or tumors. In such a case, they should be excluded from group means. 


\section{Screening of pharmaceuticals: Critical considerations}

The relevance of the results obtained from animal carcinogenicity studies in assessing human safety are most often a cause for debate. Observation of a tumorigenic response to a drug in an animal does not mean risk for humans. Factors to consider here are duration of treatment, dose, and mechanism. However, lack of a carcinogenic response in animals does not rule out a risk for humans. Further research may be needed, investigating the mode of action, which may result in confirming the presence or the lack of carcinogenic potential for humans. Mechanistic studies are useful to evaluate the relevance of tumour findings in animals for human safety.

Mechanistic studies

Mechanistic studies are often useful in the interpretation of tumour findings and in providing perspective on their relevance to human risk assessment. The choice of investigation mostly depends on the properties of the drug and/or the specific results obtained from long-term carcinogenicity testing. ${ }^{[68]}$ Short or medium-term rodent models (See section "Short or medium-term [alternative] models in carcinogenicity bioassay”) providing mechanistic insight into carcinogenic endpoints are also used. It is still acceptable to conduct a long-term carcinogenicity study in a second rodent species (i.e., mice). Changes at cellular level are studied by morphological and histochemical analyses of relevant tissues. Dose response relations for the induction of apoptosis, cell proliferation, liver foci, and changes in intercellular communication can provide better information for interpretation. ${ }^{[0]}$ Depending on the mode of action of the test substance it may be necessary to measure the levels of prolactin, thyroid-stimulating hormone, luteinizing hormone, 17ß-estradiol, gastrin, cholecystokinin, $\alpha 2 \mu$-globulin, growth factors, tissue enzyme activity, etc. A sex-specific hormone imbalance, which can be compensated, at least in part, can be examined in a separate study. ${ }^{\text {171] }}$

\section{Additional genotoxicity testing}

Compounds that are negative in the standard battery of genotoxicity tests, and lack epigenetic mechanism but are shown to be carcinogenic should be further tested for genotoxicity in appropriate models. Additional testing should include modified conditions such as metabolic activation in in vitro tests. Tests such as unscheduled DNA synthesis (UDS), ${ }^{32}$ P-postlabelling, single and double strand DNA breaks (comet assay), and mutation induction in transgenes should be included in additional testing. A candidate drug that was negative in the standard battery of tests has been demonstrated to induce DNA damage in the liver of female rats (comet assay); consequently its development was discontinued. ${ }^{\left[{ }^{[2]}\right.}$ Although the comet assay is not included in the standard battery for genotoxicity testing, it can still provide additional information. Additionally, in vivo tests measuring genotoxic damage in target organs of tumour induction may be included.

\section{Modified protocols}

In certain circumstances, it is generally encouraged to develop modified protocols that may clarify the mode of action of the test substance. Such protocols might explore the possibility of the consequence of interrupted dose regimens or the reversibility of cellular changes after cessation of dosing. For example, when dexfenfluramine (DF) was administered for 12 months in rats in long-term carcinogenicity study, plasma levels of DF were approximately two-fold higher in females than in males. When the dose was reduced to $33 \%$, the levels of DF were similar in both sexes at the end of the study. ${ }^{13]}$ Considering the pharmacokinetic/pharmacodynamic nature of the compound, a change in the design of experimental protocol can help to reevaluate the carcinogenic risk of the chemical under investigation.

\section{Advantages and limitations of lifetime carcinogenicity bioassays}

In utilizing the whole animal bioassay to identify potential cancer hazards, both dose and interspecies extrapolation can be made. In the lifetime carcinogenic bioassay, most of the carcinogenic agents that induce tumors through genotoxic mechanisms can be detected. Agents, which produce metabolites and thereby react with the DNA forming mutagenic DNA adducts and trans-species carcinogens, can be detected in long-term rodent bioassays. Within the current guidelines and testing practices, some degree of flexibility exists in designing a study that is best for a given compound. Selection is possible for rodent strains, housing conditions, group numbers above a minimum, dose levels, route of administration, diagnostic criteria, investigative techniques, types of tissues preserved for microscopic examination, data analysis, and presentation within a particular study.

Sometimes there is no clear justification for the choice of species, strain, doses, route of exposure, and time course of administration. The mechanism of action and differences in metabolism of a test substance are rarely established in an investigation. ${ }^{[74]}$ Some of the tumors in rodents arise at odd sites and/or with an unusual history complicating extrapolation of animal data to humans for a particular cancer. Many compounds are carcinogenic only at doses at or near the "maximal tolerated dose". It is sometimes unlikely and often uncertain whether the effects at MTD can justifiably be extrapolated linearly downward on the dose/response curve. The high dose may exceed the capacity of host detoxification and other defense mechanisms that would be protective at lower exposures. Some carcinogenic responses that would occur in rodents at high doses would not be likely to occur at human therapeutic exposure levels.

It is noteworthy that results for chemical carcinogenicity testing are concordant only at $70 \%$ between rats and mice. It is unlikely that the concordance between rodents and humans would be higher than that between rats and mice. There are certain tumour responses in specific organs in rodents that seem to have no counterpart in humans. The best example is the development of nephropathy and tumour in the kidneys of male rats through induction of $\alpha_{2}$-euglobulin and D-limonene. No such response occurs in female rats or in other species. ${ }^{[75]}$ Chronic progressive nephropathy, a spontaneous age-related renal disease that occurs in both sexes of rat is a risk factor for renal tumour development. However, this has no relevance for extrapolation in human risk assessment. ${ }^{[6]}$

Because the long-term bioassays are time consuming and expensive, questions are continuing to be raised on the rel- 
evance of some of the rodent responses in predicting human risk. A large body of evidence suggests that certain carcinogenic effects or mechanisms may be specific to a particular rat or mouse strain and if so, then that result should not be extrapolated to humans.

\section{Short- or medium-term (alternative) models in carcinogenicity bioassays}

One of the most important approaches in the testing of a pharmaceutical for carcinogenicity is to use a new alternative in vivo model in place of a second 2-year bioassay. The new assay should provide information that is not readily available from the lifetime bioassay and it should be able to address issues relevant to the particular pharmaceutical. [Table 2] The alternative models should supplement the long-term study and provide additional information that is not readily available from the long-term assay. These models can also be used as an additional component in the assessment of potential genotoxic carcinogenicity. But the outcome from these studies should not be considered as the decisive factor in the assessment of genotoxicity. Presently, there are no universally accepted assays identified but many hold promise and likely exhibit utility in specific circumstances. ${ }^{[77]}$ The guidelines also foster the development of assays that can provide information about the mechanism of tumor formation in response to treatment with a specific pharmaceutical. ${ }^{[1]}$

There are four short- or medium-term transgenic models in common use. The genetic changes that are relevant to the carcinogenic process are incorporated in the following models.

- Activated oncogene (Tg.AC model; rasH2 model)

- Inactivated tumour suppressor gene (p53+/- model)

- Inactivated DNA-repair gene (XPA -/- model)

These and other models have been extensively discussed in a special issue of Toxicologic Pathology, Volume 29, No-5 (Supplement), September 2001.

\section{Compound selection}

The compound selection process is to include chemicals that are representative of a broad range of mechanism, including known human genotoxic carcinogens, as well as nongenotoxic carcinogens. In addition, a large class of compounds that produce tumors in rodent by various mechanisms but not generally considered as a human hazard is also included. The

Table 2

Comparison of features/properties between traditional 2-year bioassay and alternative models for carcinogenicity testing

Features/Properties Lifetime carcinogenesis bioassay Short/medium term models

(Rats and mice)

(Transgenic and neonatal)

\section{Animals/Chemicals/Time}

Background tumour incidences

Dose

Biological variables

Mechanism of action of chemicals

Mechanism of tumorigenesis

Effect on target organ

Specificity/Sensitivity/Cost

False positive/False negative

Extrapolation to humans
Large number of animals ( $n=50$ to $100 /$ sex/group) and quantity of chemicals. 2 years

Background tumour incidences (historical value) are very high with large variables. Influence of age, sex, and strains is generally observed.

Compounds are carcinogenic only at the "maximum tolerated doses" (MTD), which is less relevant for a clinical situation.

Large variables existing between species, strains, doses, routes and duration of exposure.

Investigation on mechanism of action and differences in metabolism are not available.

Not possible to distinguish between genotoxic and epigenetic basis of tumour formation.

Sometimes target-organ specific response is not available.

Less specificity and sensitivity with huge amount of cost.

Very high rate of false positive and false negative responses.

Some tumors arise at odd sites with unusual histology as compared to humans. Extrapolation is difficult.
Less animals ( $n=15-20 /$ sex/group) and chemicals. About 6 months

The overall spontaneous tumour incidences are less with histopathological consistency. No influence of age on tumour formation.

Dose dependent increase of tumour incidence provides supportive evidence that the tumors are the result of chemicals administered.

Fewer variables with genetically homogenous population are considered for the study.

Mechanism of action at molecular level can be envisaged and possible to differentiate between different genotoxic events.

Distinguish between genotoxic and epigenetic basis of tumour formation.

Mostly target-organ specific response in tumour induction is obtained.

High specificity and sensitivity with moderate cost.

Less chance of false positive and false negative tumour responses.

Mechanistic basis of tumour induction enhances confidence in extrapolation. 
compounds, which are selected for investigation, already have comprehensive existing databases of toxicological information.

\section{Tg.AC transgenic mice model}

In these animals, four copies of the $\mathrm{V}$-Ha-ras oncogene fused to fetal zeta globin promoter of chromosome 11 of strain FVB/N mice are inserted. There are hemizygous and homozygous types, but the later appear to respond more consistently. ${ }^{178 \mid}$ Topical application of carcinogens to the shaved dorsal surface of Tg.AC mice induces epidermal squamous cell papillomas or carcinomas, a reporter phenotype that defines the activity of the chemical. ${ }^{[79]-[81]}$ The oral route of administration can also generate tumorigenic response in Tg.AC mice and result in squamous cell papillomas or carcinomas of the fore stomach. ${ }^{[22]}$ Animals are exposed on dermal surface for 20 weeks and observed for an additional 6 weeks for the appearance of skin papillomas. This model is good for detecting mutagenic and non-mutagenic carcinogens, including tumour promoters but unlikely to detect certain carcinogens, that are strain or species-specific in the conventional 2-year bioassay. ${ }^{[83]}$

\section{Tgras H2 transgenic model}

In this model, the mouse strain of CB6F1 carries five or six copies of the human $c$-Ha-ras oncogene per cell with its own promoter/enhancer. The rasH2 mouse is a hemizygous strain because the homozygous state is lethal. ${ }^{[8]}$ The transgene is constructed by ligating human activated $c$-Ha-ras gene with single point mutations at codon 12 and 6185. The tumors in hemizygous transgenic mice are rare until 6 months of age. In the rasH2 mouse, point mutations of the transgene induced by genotoxins are reported frequently, but not in all tumors. Elevated level of transgene expression is detected in all genotoxin-induced tumors in the rasH2. The rasH2 model responds to a spectrum of weakly to strongly genotoxic compounds and may be useful in the detection of compounds of concern as a risk for human cancer. The rasH2 mouse model appears to have greater sensitivity than non-transgenic mice to compounds that are human carcinogens but are nonmutagenic in the Salmonella assay. Furthermore, the rasH2 model does not appear to be susceptible to compounds that are rodent carcinogens because it operates through nongenotoxic mechanisms for carcinogenesis.

\section{The p53 +/- knockout mice model}

Mice with a single p53 allele have been considered analogous to humans at risk for heritable forms of cancer such as the Li-Fraumeni syndrome. ${ }^{[86]}$ The accumulated data indicate that p53+/- mice are generally sensitive to known genotoxic carcinogens within six months time frame, but are considered insensitive to non-genotoxic carcinogens. ${ }^{\text {[87|-[89] }}$ The carcinogenesis in the p53 mouse model is likely due to the carcinogen-tissue interactions, the mutation in oncogenes, the affect in the cell signaling pathways and the duration in latency period in tumour formation. On the basis of extensive data and sensitivity to carcinogen it has been proposed that the 6month p53 +/- carcinogenicity assay could substitute for one of the 2-year rodent (preferably mice) bioassays currently required for pharmaceutical licensing by the US FDA. ${ }^{.90]}$

The XPA-/- knockout mice

In this model, C57BIL/6 mice have a deletion across exons
3 and 4 of both XPA alleles, rendering the cells totally defective in nucleotide excision DNA repair. Therefore, the animals are comparable with human repair-deficient cancer-prone conditions such as xeroderma pigmentosum (XP). As in humans with XP, the Xpa deficient mice develop skin cancer at UVexposed areas of the body. Squamous cell carcinomas (SCCs) are found in the eyes and skin of Xpa- deficient mice. ${ }^{\text {[91], [92] The }}$ Xpa-deficient mice are sensitive to genotoxic carcinogens that are reactive to the nucleotide excision repair (NER) pathway. The tumors appear within an exposure time of 9 months. From the results, it is evident that the Xpa mice mimic the phenotype of humans with XP and that these mice could be used to identify human carcinogens.

\section{The neonatal mouse model}

The mouse is treated with test substance at various time points after its birth and up to 3 weeks of age. This model is considered sensitive for the detection of carcinogens that operate via a genotoxic mode of action. It doesn't respond to chemicals that act via epigenetic mechanisms, which are commonly evaluated in 2-year carcinogenicity studies. As such, the model has a high sensitivity and specificity in its response. ${ }^{[93], ~[94]}$ The general advantages of this model are: lesser amount of test compound, few animals, faster completion time, and low cost in operation. The neonatal mouse assay has a sensitivity of $85 \%$ and a positive prediction rate of $96 \%$ in comparison to 2-year carcinogenicity bioassay. ${ }^{[95]}$ A positive result in the neonatal assay indicates that a compound is likely to be a trans-species genotoxic carcinogen. Negative data will contribute to the weight of evidence that a tumour response observed in the rat is more likely to involve an epigenetic mode of action.

\section{Evaluation of pharmaceuticals across the models}

\section{Nongenotoxic and noncarcinogens}

The evaluation of rodent noncarcinogens in alternative models depends on the specificity and sensitivity of a test substance toward a model. Three non-genotoxic chemicals, ampicillin, d-mannitol, and sulfisoxazole are demonstrated noncarcinogens in all in vivo transgenic models. ${ }^{[96]}$ It may be noted that these animals' genetic constructs were specifically designed to increase the susceptibility to carcinogens. The negative results obtained across the models suggest that these alternative models are not over sensitive owing to either the insertion or deletion of the genes. These drugs are still considered noncarcinogenic to humans in spite of the lack of specific epidemiological data.

\section{Genotoxic carcinogens}

Three genotoxic chemicals that are known to be carcinogens in 2-year bioassay in rodents and in humans were evaluated in short-term models. The chemicals included cancer chemotherapeutic agents, cyclophosphamide, melphalan, and the analgesic, phenacetin. Cyclophosphamide and melphalan were positive in all of the short-term models evaluated except for the Tg.AC dermal exposure model, in which the results were determined to be equivocal. In contrast, phenacetin was negative in all models except the TgrasH2 model. Generally, all these models have the ability to detect genotoxic compounds or their metabolites, if they interact directly with the DNA. Phenacetin is a weak genotoxic as evident from several 
genotoxicity studies. In the mouse, it produced tumors in renal and urothelial cells, ${ }^{[97]}$ whereas in humans, at a very high dose, it was carcinogenic for the urothelium, particularly the renal pelvis. ${ }^{[98]}$ It presents some difficulties when interpreting the relation between genotoxicity and carcinogenicity for genotoxic carcinogens in various animal models. The positive results in the TgrasH2 model suggest that this model might be suitable for evaluating weakly genotoxic chemicals.

\section{Immunosuppressant and hormonal carcinogens}

Cyclosporin A, a pharmaceutical used clinically as an immunosuppressant, is non-genotoxic and was shown to be negative in the 2-year rat bioassay. ${ }^{[96]}$ However, in humans it is clearly associated with an increased risk of producing various tumors. Therefore, it is classified as a non-genotoxic, known human carcinogen. It was also tested positive in the p53+/and Xpa-/- mouse models and the dermal Tg.AC assay. It gave equivocal results in the oral Tg.AC and Tgras $\mathrm{H} 2$ models and was negative in the neonatal mouse model. The observation that cyclosporin A produces positive results in most of these transgenic and knockout models demonstrate that tumour can be produced in these models without the chemical being genotoxic. Two estrogenic compounds, diethylstilbestrol (DES) and estradiol were evaluated recently for their carcinogenic potential in short-term models. DES is carcinogenic in animals and acts primarily through stimulation of increased cell proliferation and by binding to estrogen receptors. Likewise, estradiol is known to increase the risk of cancer through the stimulation of increased cell proliferation. Now there is an evidence to show that estradiol is a weak genotoxic and might form DNA adduct. ${ }^{[99]}$ Estradiol was demonstrated positive in the dermal Tg.Ac, Хpa, and p53 mouse models. However, it was negative in the oral Tg.Ac, TgrasH2, and Xpa, and equivocal in the p53 models. On the other hand, DES was demonstrated positive in all of the in vivo mouse models except that of oral Tg.AC and neonatal mouse models.

Non-genotoxic rodent carcinogens, putative human noncarcinogens

Several non-genotoxic pharmaceuticals that were found to be carcinogenic in one or more long-term rodent bioassays were evaluated on alternative models for carcinogenicity assessment. On the basis of either on the epidemiological evaluation or on mechanistic considerations, these chemicals have not been considered to pose a carcinogenic hazard in humans. The chemicals included in this category were phenobarbital, methapyrilene, reserpine, dieldrin, haloperidol, chlorpromazine, chloroform, metaproterenol, and sulphamethoxazole. All of them were negative in all models, except for an equivocal result for chloroform in the p53 model. ${ }^{[96]}$ On the other hand, clofibrate, diethylhexylphthalate (DEHP), and WY- 14643 (all are peroxisome proliferators), which are non-genotoxic and produce tumors in rodent bioassays, however, are putatively non carcinogenic in humans gave widely variable results in short-term models. ${ }^{[96]}$ The reason for this variability is unknown, but indicates that there is not a complete correlation between direct DNA reactivity (genotoxicity) and positivity in these transgenic models. ${ }^{[00]}$ It further suggests that the alternative models do not have $100 \%$ specificity in distinguishing between human carcinogens and noncarcinogens or rodent carcinogens.

\section{Usefulness and limitations of alternative models}

Pharmaceutical companies have already started using these models for carcinogenicity testing of pharmaceuticals as a result of the discussion of International Conference on Harmonization in 1995. ${ }^{\text {[101] }}$ The details of these limitations and usefulness are provided in Table 2. It is apparent that these models are useful for screening purposes in a regulatory context with respect to potential carcinogenic hazard to humans. ${ }^{[102]}$ They have the advantage of requiring a shorter time (6 months versus 2 years), fewer animals (15-25 versus 50-100 animals/sex/dose level), and less cost. It can give further insight into the mechanistic aspects of tumorigenesis, which operates at cellular and/or molecular level. However, the sensitivity and specificity suggest that these models are not definitive determinants of potential cancer risk even when applied to a limited group of chemicals that have relatively well-defined properties. ${ }^{[103]}$ Using these models to evaluate chemicals with unknown carcinogenic potential and having varying biological properties pose even greater difficulties. One of the major limitations of these models is the development of an increased incidence of spontaneous tumors in these respective strains. ${ }^{[89],[104]}$ The spontaneous tumors in mice generally do not have direct relevance to human situation. Again, they appear to have limited usefulness in predicting target-organ specificity for human carcinogens, because positive results are generally obtained at the highest dose (MTD). ${ }^{[105], ~[106] ~}$ These models are less sensitive to non-genotoxic chemicals that are carcinogenic in the traditional 2-year bioassay, and are generally considered non carcinogenic to humans on the basis of their mechanism and/or epidemiology data.

\section{In Silico prediction}

The goal of in silico prediction is to develop software-based structure-activity relation (SAR) models, which can predict the carcinogenic potential of pharmaceuticals. SAR has been applied for the recognition of structural alerts present in the chemicals and to understand their interactions with living organisms. The quantitative structure activity relationship (QSAR) is determined to build mathematical models linking the physicochemical properties of the molecules to the toxicological end-points. ${ }^{[107]}$ The MDL QSAR (formerly SciVision QSAR) model is used to predict carcinogenic potential of wellvalidated compounds for which a large database already exists in the literature. The true prediction value of these models is to decrease the risk of unexpected results that would appear very late in the development process and in life time carcinogenesis bioassays. ${ }^{[108]}$ These prediction models can facilitate the design of new compounds with potentially improved properties and may also shed light on the underlying aspects of mechanism of action of carcinogens. The informatics and computational safety analysis division of FDA's Centre for Drug Evaluation and Research (CDER) uses in silico prediction models for the prediction of carcinogenic potential in regulatory and scientific decision making. The in silico method of carcinogenicity prediction is further restricted by many factors including the complexity of carcinogenic mechanism, the 
noncongeneric nature of study compounds, and the limitations and uncertainty inherent in the design, and interpretation of 2-year rodent carcinogenicity studies. ${ }^{[109]}$ It is extremely difficult to create computerized methods that can incorporate all of the factors including biological modalities and idiosyncrasies that must be considered when predicting carcinogenicity of chemicals. With the introduction of bioinformatics, data mining techniques and mechanistic studies can further provide many technology platforms, data interpretation, and regulatory perspectives in carcinogenic risk estimation in forecasting human carcinogenic potential of chemicals. ${ }^{[10], ~[111] ~}$

\section{Future of carcinogenesis bioassays}

Carcinogenesis is a complex process involving multiple factors. Owing to several inherent limitations in lifetime rodent carcinogenesis bioassay, extrapolation of the results to humans is difficult. In order to reduce the uncertainties and to render the extrapolation (of animal findings to man) more reliable, various mechanistic end-points have to be explored for a particular study. It is necessary to combine the results of these assays with the results from short-term tests, toxicokinetics, pharmacodynamics, genetic variation, and structure-activity relation. The introduction of new short-term models and the understanding of mechanistic aspects can greatly facilitate not only cancer research but also regulatory aspects of drug safety evaluation. With the introduction of ICH guidelines, efforts are now underway to validate these models for regulatory drug safety evaluation. The guidelines aim to be flexible for a new group of pharmaceuticals, especially biotechnology derived products. By adopting the ICH guidelines, the process of new drug approval by the regulatory authorities can be streamlined, and reduce the time and conserve the resources in drug discovery and development program. Pharmaceutical companies and regulatory agencies have to prepare themselves to implement and adopt these new techniques to meet the challenges of the $21^{\text {st }}$ century. In a developing country like India, the existing guidelines need to be reevaluated and modified in accordance to newer trends of globalization.

\section{Concluding remarks}

The transgenic models could be used in place of traditional long-term bioassay in mice, and in conjunction with a standard 2-year bioassay in the rat. However, these models cannot be used in isolation when determining either genotoxicity or potential carcinogenicity in humans. The screening tests need to be sensitive enough to minimize false negatives, and specific enough to reduce false positives so that the development prospects of a new drug are not hindered. These in vivo models have potential usefulness as screening bioassays for detecting potential carcinogenic hazards of pharmaceuticals in humans. However, like other models, these new alternative bioassays do not accurately predict with $100 \%$ sensitivity or specificity in evaluation of carcinogenic potential to humans. Owing to rapid synthesis of chemicals, and increasing speed and efficacy of toxicological assessment by pharmaceutical companies, regulatory agencies are preparing themselves to meet the challenges of the $21^{\text {st }}$ century by reevaluating the existing protocols in the changing environment. In a developing country like India, the existing guidelines need to be reevaluated and modified in accordance with the newer trends of globalization but without sacrificing the quality and the safety of medicines.

\section{References}

1. Van der Laan JW. Current status and use of short/medium-term models for assessment of carcinogenicity of human pharmaceuticals: Regulatory perspectives. Toxicol Lett 2000;112-113:567-72.

2. McConnel EE, Solleveld HA, Swenberg JA, Boorman GA. Guidelines for combining neoplasms for evaluation of rodent carcinogenesis studies. J Natl Cancer Inst 1986;76:283-9.

3. Rushton B. Oncogenicity/carcinogenicity. In: Cartwright AC, Mathews BR, editors. International Pharmaceutical Product Registration: Aspects of quality, safety and efficacy. New York: Ellis Horwood.1994.

4. Huff J. Value, validity and historical development of carcinogenesis studies for predicting and confirming carcinogenic risks to humans. In: Carcinogenicity testing, predicting and interpreting chemical effects. New York: Marcel Dekker; 1999.

5. Federal Register Part II Office of Science and Technology Policy. Chemical Carcinogens: A Review of the Science and its Associated Principles. 1985.

6. Ashby J, Doerrer NG, Flamm FG, Harris JE, Hughes DH, Johannsen FR, et al. New findings relate aminoglycoside ototoxicity to cumulative plasma or perilymph AUC. Infect Dis News Lett 1990;11:4-6.

7. Snyder RD, Green JW. A review of the genotoxicity of marketed pharmaceuticals. Mutat Res 2001:488:151-69.

8. Haseman JK, Boorman GA, Huff J. Value of historical control data and other issues related to the evaluation of long-term rodent carcinogenicity studies. Toxicol Pathol 1997;25:524-7.

9. Nath J, Krishna, G. Safety screening of drugs in cancer therapy. Acta Haematol 1998;99:138-47.

10. Trosko JE. Challenge to the simple paradigm that 'carcinogens' are 'mutagens' and to the in vitro and in vivo assays used to test the paradigm. Mutat Res 1997;373:245-9.

11. Yuspa SH. Overview of carcinogenesis: past, present and future. Carcinogenesis 2000;21:341-4.

12. Hanawalt PC. Genomic instability: Environmental invasion and the enemies within. Mutat Res 1998;400:117-25.

13. Huff J, Haseman J, Rall D. Scientific concepts, value and significance of chemical carcinogenesis studies. Ann Rev Pharmacol Toxicol 1991;31:62152.

14. Tennant RW. The genetic toxicity database of the National Toxicology Program: Evaluation of the relationships between genetic toxicity and carcinogenicity. Environ Health Perspect 1991;96:47-51.

15. Vogelstein B, Kinzler KW. The multi-step nature of cancer. Trend Genet 1993:9:138-41.

16. Perera FP. Molecular epidemiology: On the path to prevention? J Natl Cancer Inst 2000;92:602-12.

17. Grubowski HG, Vernon JM. Returns to R \& D on new drug introductions in the 1980s. J Health Econ 1994;13:383-406.

18. Ulrich R, Friend SH. Toxicogenomics and drug discovery: Will new technologies help us produce better drugs? Nat Rev 2002;1:84-8.

19. Weisburger JH. Carcinogenicity and mutagenicity testing, then and now. Mutat Res 1999;437:105-12.

20. Satyavati GV. Principles for testing carcinogenic effects of drugs and chemicals. Indian J Pharmacol 1972;4:57-64.

21. Marathe M, Thomas GP. Current status of carcinogenicity evaluation in drug research. Indian J Pharmacol 1985;17:120-26.

22. Scales MDC, Griffin J. Use of two matched strains: Can they improve sensitivity? In: McAuslane JAN, Lumley CE, Walker SR, editors. The carcinogenicity debate. London: Quay Publishing Limited; 1992.

23. Spielmann $\mathrm{H}$, Liebsch $\mathrm{M}$. Lessons learned from validation of in vitro toxicity test: From failure to acceptance into regulatory practice. Toxicol in vitro 2001;15:585-90.

24. Berend K. Good laboratory practice in the European community. Role of the commission and the member states: External aspects. Ann Ist Super Sanita 2002;38:9-13.

25. Drugs and Cosmetic Act. Requirements and guidelines on clinical trials for import and manufacture of new drugs. In: Malik V, editor. Drugs and Cosmetic Act. 1940, $12^{\text {th }}$ ed. Lucknow: Eastern Book Company; 1999. p. 394-404.

26. EC Pharmaceuticals Council recommendation 83/571/EEC, Annex 1- Repeated dose toxicity. Off J Euro Commun 1983;332:12-7. 
27. Japan Pharmaceuticals. Guidelines for toxicity studies of drugs. Pharmaceutical Affairs Bureau, Ministry of Health and Welfare. Tokyo: Nippo Limited; 1989.

28. Torok G, Csik M, Pinter A, Surjan A. Carcinogenicity of medicinal drugs: A survey of IARC monographs. Euro J Public Health 2000;8:83-5.

29. Huff J. IARC monographs, industry influence, and upgrading, downgrading and under grading chemicals. Int J Occup Environ Health 2002;8:249-355.

30. Robinson DE, Macdonald JS. Background and framework for ILSI's collaborative evaluation program on alternative models for carcinogenicity assessment. Toxicol Pathol 2001;29:13-9.

31. Sistare FD, Thompson KL, Honchel R, Degeorge J. Evaluation of the Tg. AC transgenic mouse assay for testing the human carcinogenic potential of pharmaceuticals-Practical pointers, mechanistic clues and new questions. Int J Toxicol 2002;21:65-79.

32. Storer RD, French JE, Haseman J, Hajian G, Legrand EK, Long GG, et al. p53+/- Hemizygous knockout mouse: Overview of available data. Toxicol Pathol 2001;29:30-50.

33. Tennant RW, Stasiewicz S, Mennear J, French JE, Spalding JW. Genetically altered mouse models for identifying carcinogens. IARC Scientific Publication 1999;146:123-50

34. Tamaoki $\mathrm{N}$. The rasH2 transgenic mouse: Nature of the model and mechanistic studies on tumorigenesis. Toxicol Pathol 2001;29:18-89.

35. Popp JA. Criteria for the evaluation of studies in transgenic models. Toxicol Pathol 2001;29:20-3.

36. Christian MS. Overview of the fourth International Conference on Harmonization. Int J Toxicol 1997;16:659-68.

37. Contrera JF, Jacobs AC, DeGeorge JJ. Carcinogenicity testing and the evaluation of regulatory requirements for pharmaceuticals. Reg Toxicol Pharmacol 1997;25:30-45.

38. Davies TS, Monro A. The rodent carcinogenicity bioassay produces a similar frequency of tumour increases and decreases: Implications for risk assessment. Reg Toxicol Pharmacol 1994;20:281-301.

39. Van Oosterhout JP, Van der Laan JW, De Waal EJ, Olejniczak K, Hilgenfeld M, Schmidt $\mathrm{V}$, et al. The utility of two rodent species in carcinogenic risk assessment of pharmaceuticals in Europe. Reg Toxicol Pharmacol 1997;25: 6-17.

40. ICH Topic S1A. Guideline on the need for carcinogenicity studies of pharmaceuticals. International Conference on Harmonization on Technical Requirements for Registration of Pharmaceuticals for Human use. Harmonized Tripartite Guideline; 1995.

41. ICH Topic S1B: Testing for carcinogenicity of pharmaceuticals: International Conference on Harmonization on Technical Requirements for Registration of PharmaceutCicals for Human use. Harmonized Tripartite Guideline; 1996.

42. Claude JR. Difficulties in conceiving and applying guidelines for the safety evaluation of biotechnologically-produced drugs: some examples. Toxicol Lett 1992;64-65:349-55

43. Gold LS, Slone TH, Ames BN. What do animal cancer tests tell us about human cancer risk? Overview of analyses of the carcinogenic potency database. Drug Metab Rev 1998;30:359-404.

44. Gollapudi BB, Krishna G. Practical aspects of mutagenicity testing strategy: an industrial perspective. Mutat Res 2000;455:21-8.

45. Jena GB, Kaul CL, Ramarao P. Genotoxicity testing, a regulatory requirement for drug discovery and development: Impact of $\mathrm{ICH}$ guidelines. Indian $\mathrm{J}$ Pharmacol 2002;34:86-99.

46. Omenn GS. Assessment of human cancer risk: Challenges for alternative approaches. Toxicol Pathol 2001;29:5-12.

47. US FDA Guidance for Industry. Carcinogenicity study protocol submission. Centre for Drug Evaluation and Research (CDER) 2002.

48. Deschl U, Kittel, B, Rittingghausen S, Morawietz G, Kohler M, Mohr U, et al. The value of historical control data- scientific advantages for pathologists, industry and agencies. Toxicol Pathol 2002;30:80-7.

49. Haseman JK. Data analysis: Statistical analysis and use of historical control data. Reg Toxicol Pharmacol 1995;21:52-9.

50. Gold LS, Manley NB, Ames BN. Extrapolation of carcinogenicity between species: Qualitative and quantitative factors. Risk Anal 1992; 12:579-88.

51. Muller L, Kasper P. Human biological relevance and the use of thresholdarguments in regulatory genotoxicity assessment: Experience with pharmaceuticals. Mutat Res 2000;418:121-9.

52. Monro A. Interspecies comparisons in toxicology: The utility and futility of plasma concentrations of the test substance. Reg Toxicol Pharmacol 1990;12:137-60.

53. Peck CC, Barr WH, Benet LZ, Collin J, Desjardins RE, Furst DE, et al. Opportunities for integration of pharmacokinetics and toxicokinetics in rational drug development. Pharm Res 1992;9:826-33.

54. Britton LD, Downes N, Mullins P, Mitchell D. The most appropriate strain for rat carcinogenicity bioassay [abstract]. Toxicol Sci 2004;78:560.

55. Roe FJC. 1200 rat bioassay study: Designed overview of results. In: Fishbein L, editor. Biological effects of dietary restriction. 1991.

56. Peto R, Pike MC, Day NE, Gray RG, Lee PN, Parish S, et al. Guideline for simple, sensitive, significant tests for carcinogenic effects in long term animal experiments. Long term and short term screening assays for carcinogens. A critical appraisal IARC Monograph Services 2. 1980.

57. Lee PN. Statistics. In: Anderson D, Conning DM, editors. Experimental toxicology the basic issues. Athenaeum Press; 1992.

58. Lin JH, Lu AYH. Role of pharmacokinetics and metabolism in drug discovery and development. Phamacol Rev 1997;49:403-48.

59. Chhabra RS, Huff JE, Schwetz BS, Selkirk J. An overview of pre-chronic and chronic toxicity/carcinogenicity experimental study designs and criteria used by the National Toxicology Program. Environ Health Perspect 1990;86:31321.

60. ICH S1C: Dose selection for carcinogenicity studies of pharmaceuticals. International Conference on Harmonization on Technical Requirements for Registration of Pharmaceuticals for Human use. Harmonized Tripartite Guideline. 1994.

61. Melnick RL, Kohn M. Dose response analyses of experimental cancer data, Drug Metab Rev 2000;32:193-209.

62. Haseman JK. Issues in carcinogenicity testing: Dose selection. Fundam Appl Toxicol 1985;5:66-78.

63. Fielder RJ, Allen JA, Boobis AR, Doe J, Esdail DJ, Gatehouse DG, et al. Report of the BTS/EKEMS working group on dose setting in in vivo mutagenicity assays. Human Exp Toxicol 1993;12:189-98.

64. Beaubien AR. New findings relate aminoglycoside ototoxicity to cumulative plasma or perilymph AUC. Infect Dis News Lett 1992;11:4-6.

65. Bergman K, Olofsson I, Sjoberg P. Dose selection for carcinogenicity studies of pharmaceuticals: systemic exposure to phenacetin at carcinogenic dose in the rat. Reg Toxicol Pharmacol 1998;28:226-9.

66. Calabrese EJ. Suitability of animal models for predictive toxicology: theoretical and practical considerations. Drug Metab Rev 1984;15:505-23.

67. Harada M, Murata J, Sakamura Y, Sakakibara H, Okuno S, Suzuki T. Carrier and dose effects on the pharmacokinetics of T-0128, a camptothecin analoguecarboxymethyl dextran conjugate, in non-tumour and tumour bearing rats. J Cont Rel 2001;71:71-86.

68. Vainio H, Magee PN, McGregor DB, McMichael AJ. Mechanisms of carcinogenesis in risk identification, IARC Scientific Publications. Lyon: Int Agen Res Can 1992;116:1-616.

69. Dixit R, Riviere J, Krishnan K, Andersen ME. Toxicokinetic and physiologically based toxicokinetics in toxicology and risk assessment. J Toxicol Environ Health 2003:6:1-40.

70. Dragan YP, Bidlack WR, Cohen SM, Goldsworthy TL, Hard GC, Howard PC, et al. Implications of apoptosis for toxicity, carcinogenicity and risk assessment: fumonisin B(1) as an example. Toxicol Sci 2001;61:6-17.

71. Gross CJ, Kramer JA. The role of investigative molecular toxicology in early stage drug development. Expert Opin Drug Safety 2003;2:147-59.

72. Van Gompel JAJ, Thilemans L, Geerts K, Vanparys P. Evaluation of the added value of the single cell gel electrophoresis test to the standard battery of genetic toxicology tests [abstract]. Environ Mol Mutagen 1999;33:65.

73. Campbell DB, Jochemsen R. Animal pharmacokinetics and toxicokinetics. In: Cartwright AC, Mathews BR, editors. International Pharmaceutical Product Registration: Aspects of quality, safety and efficacy. New York: Ellis Horwood; 1994.

74. Weisburger JH, Williams GM. Carcinogen testing: Current problems and new approaches. Science 1981;214:401-7.

75. McClain RM. Mechanistic considerations in the regulation and classification of chemical carcinogens. In: Kotsonis F, Mackey M, Hijele J, editors. Nutritional Toxicology. New York: Raven;1994.

76. Hard GC, Khan KN. A contemporary overview of chronic progressive nephropathy in the laboratory rat, and its significance for human risk assessment. Toxicol Pathol 2004;32:171-80.

77. Goodman Jl. A perspective on current and future uses of alternative models for carcinogenicity testing. Toxicol Pathol 2001;29:173-6.

78. Eastin WC, Mennear JH, Tennant RW, Stoll RE, Branstetter DG, Bucher JR, et al. Tg.AC genetically altered mouse: Assay working group overview of available data. Toxicol Pathol 2001;29:60-80. 
79. Spalding JW, French JE, Tice RR, Furedi-Machacek M, Haseman JK, Tennant RW. Development of a transgenic mouse model for bioassays: Evaluation of chemically induced skin tumors in Tg.AC mice. Toxicol Sci 1999;49:241-54.

80. Tennant RW, French JE, Spalding JW. Identifying chemical carcinogens and assessing potential risk in short-term bioassays using transgenic mouse models. Environ Health Perspect 1995;103:942-50.

81. Tremus CS, Mahler JF, Anthaswamy HN, Loughlin SM, French JE, Tennant RW. Photocarcinogenesis and susceptibility to UV radiation in the V-Ha-ras transgenic Tg.AC mouse. J Invest Dermatol 1998;111:445-51.

82. Cannon RE, Graves S, Spalding JW, Trempus CS, Tennant W. Oral administration of dimethyl vinyl chloride increases frequency of forestomach papillomas in Tg.AC mice. Molec Carcin 2000;29:229-35.

83. Tennant RW, Stasiewicz S, Eastin WC, Mennear JH, Spalding JW. The Tg.AC (v-Ha-ras) Transgenic mouse: Nature of the model. Toxicol Pathol 2001;29: 51-9.

84. Saitoh A, Kimura M, Takahashi R, Yokoyama M, Nomura T, Izawa M Sekiya T, et al. Most tumors in transgenic mice with human c-Ha-ras gene contained somatically activated transgenes. Oncogene 1990;5:1195-200.

85. Sekiya T. Prassolov VS, Fushimi M, Nishimura S. Transforming activity of the c-Ha-ras oncogene having two point mutations in the codon 12 and 61. Japan J Cancer Res 1985;76:851-5.

86. Malkin D, Li FP, Strong SC, Fraumeni JF Jr, Nelson CE, Kim DH, et al. Germline p53 mutations in a familial syndrome of breast cancer, sarcomas and other neoplasms. Science 1990;250:1233-8.

87. Spalding JW, French JE, Stasiewicz S, Furedi-Machacek M, Connor F, Tice $\mathrm{RR}$, et al. Responses of transgenic mouse lines p53(+/-) and Tg.Ac to agents tested in conventional carcinogenicity bioassays. Toxicol Sci 2000;53:213-23.

88. Tennant RW, Spalding J, French JE. Evaluation of transgenic mouse bioassays for identifying carcinogens and noncarcinogens. Mutat Res 1999;365:119-27.

89. Gulezian D, Jacobson-Ram D, McCullough CB, Olson H, Recio L, Robinson $\mathrm{D}$, et al. Use of transgenic animals for carcinogenicity testing: Considerations and implications for risk assessment. Toxicol Pathol 2000;28:482-99.

90. Contrera JF, DeGeorge JJ. In vivo transgenic bioassays and assessment of the carcinogenic potential of pharmaceuticals. Environ Health Perspect 1998; 106:71-80.

91. De Vries A, Van Oostrom CTHM, Hofhuis FMA, Dortant PM, Berg RJW, De Gruijl FR, et al. Increased susceptibility to ultraviolet-B and carcinogens of mice lacking the DNA excision repair gene XPA. Nature 1995;377:169-73.

92. De Vries A, Gorgels TG, Berg RJW, Jansen GH, Van Steeg H. Ultraviolet-B induced huperplasia and squamous cell carcinomas in the cornea of XPAdeficient mice. Exp Eye Res 1998;67:53-9.

93. Flammang TJ, Von Tungeln LS, Kadlubar FF, Fu PP. Neonatal mouse assay for tumourigenicity. Alternative to the chronic rodent bioassay. Reg Toxicol
Pharmacol 1997;26:230-40.

94. Fu PP, Von Tungeln LS, Yi P, Xia Q. Neonatal mouse tumorigenicity bioassay. Drug Information J 1998;32:711-28.

95. Fuji K. Evaluation of the newborn mouse model for chemical tumorigenesis. Carcinogenesis 1991;12:1409-15.

96. Cohen SM. Alternative models for carcinogenicity testing: Weight of evidence evaluation across models. Toxicol Pathol 2001;29:183-90.

97. Nakanishi K, Kurata Y, Oshima M, Fukushima S, Ito N. Carcinogenicity of phenacetin: Long-term feeding study in B6C3F1. Int J Cancer 1982;29:439-44.

98. Johansson SL, Angervall L, Bengtsson U, Wahlqvist L. Uroepithelial tumors of the renal pelvis associated with abuse of phenacetin-containing analgesics. Cancer 1974;33:743-53.

99. Carmichael PL, Mills JJ, Campbell M, Basu M, Caldwell J. Mechanism of hormonal carcinogenesis in the p53+/- hemizygous knockout mouse: Studies with diethylstilbestrol. Toxicol Pathol 2001;29:155-60.

100. Rosenkranz HS. Synergy between systemic toxicity and genotoxicity: Relevance to human cancer risk. Mutat Res 2003;529:117-27.

101. International Conference on Harmonization (ICH). Technical requirements for registration of pharmaceuticals for human use. Federal Registrar 1995;60:11278-86.

102. Ashby J. Expectations for transgenic rodent cancer bioassay models. Toxicol Pathol 2001;29:177-82.

103. Cohen SM, Robinson D, MacDonald J. Alternative models for carcinogenicity testing. Toxicol Sci 2001;64:14-9.

104. Maronpot RR, Boorman GA. The contribution of the mouse in hazard identification studies. Toxicol Pathol 1996; 24:726- 31.

105. Foran JA. Principles for the selection of doses in chronic rodent bioassays. Washington, DC: ILSI Press; 1997.

106. Swenberg JA. Bioassay design and MTD setting: Old methods and new approaches. Reg Toxicol Pharmacol 1995;21:44-51.

107. Benigni R, Zito R. The second National Toxicology Program comparative exercise on the prediction of rodent carcinogenicity: definitive results. Muta Res 2004;566:49-63.

108. Louis JC. Predicting carcinogenicity early: The latest in silico solution. Biosilico 2003;1:115-6.

109. Contrera JF, Mathews EJ, Benz RD. Predicting the carcinogenic potential of pharmaceuticals in rodents using molecular structural similarity and E-state indices. Reg Toxicol Pharmacol 2003;38:243-59.

110. Suter L, Babiss LE, Wheeldon EB. Toxicogenomics in predictive toxicology in drug development. Chem Biol 2004;11:161-71.

111. Gottmann E, Kramer S, Pfahringer B, Helma C. Data quality in predictive toxicology: Reproducibility of rodent carcinogenicity experiments. Environ Health Perspect 2001;109:509-14.

\title{
$51^{\text {th }}$ ANNUAL CONFERENCE OF THE ASSOCIATION OF PHYSIOLOGISTS AND PHARMACOLOGISTS OF INDIA
}

\section{Date : December $12-15^{\text {th }}, 2005$ \\ Venue : JIPMER, Pondicherry}

\section{Contact:}

\author{
Dr. MADANMOHAN, T. \\ Organizing Secretary, APPICON 2005 \\ Department of Physiology, JIPMER, Pondicherry - 605006. \\ E-mail: drmadanmohan123@rediffmail.com; appicon2005@gmail.com
}

